\title{
A Tool for Beamforming and Real-Time Link Budget Analysis in Aeronautical Communications Using Kinematics
}

\author{
M. Cenk Erturk ${ }^{1,2}$ and Yasin Aksan ${ }^{1,3}$ \\ ${ }^{1}$ Meteksan Defense Inc., Ankara, Turkey \\ ${ }^{2}$ Department of Electrical Engineering, University of South Florida, Tampa, FL 33620, USA \\ ${ }^{3}$ Department of Electrical \& Electronics Engineering, TOBB University of Economics \& Technology, Ankara, Turkey
}

Correspondence should be addressed to M. Cenk Erturk; certurk@meteksan.com

Received 31 March 2016; Revised 15 June 2016; Accepted 10 July 2016

Academic Editor: Fun $\mathrm{Hu}$

Copyright ( 2016 M. C. Erturk and Y. Aksan. This is an open access article distributed under the Creative Commons Attribution License, which permits unrestricted use, distribution, and reproduction in any medium, provided the original work is properly cited.

\begin{abstract}
Aeronautical Communication (AC) systems are likely to be a part of future tiered communication network structures. Therefore maintaining a robust AC link with a minimum power burden on the host platform has a critical importance. In this paper, we analyze the AC systems from a link budget analysis point of view and define the requirements for the parameters of link budget with an emphasize on antenna gains. First, we study the link budget analysis in an AC system. Then, we present a mathematical framework to provide an end-to-end link budget analysis utilizing the platform kinematics. Finally, we present the numerical results for typical AC scenarios and discuss that these results can be used for calculating the real-time link budget and electronic beamforming to provide a robust link.
\end{abstract}

\section{Introduction and Motivation}

Aeronautical communications (AC) is an emerging area in which aeronautical platforms are considered as a part of the multitier network for future wireless communication systems. Programs led by the National Aeronautics and Space Administration (NASA), the Federal Aviation Administration (FAA), and EUROCONTROL all include the aeronautical platforms as part of the multitier network $[1,2]$. The driving reasons for development of high data rate AC systems are as follows: (1) the increase in data demand for Air Traffic Control and Air Traffic Management due to the growth in air transportation [3], (2) the need for low latency and low cost services to provide in-flight multimedia access [4], (3) the potential to use AC systems as a backbone for terrestrial communication networks [5], and (4) the exponential increase in the number of unmanned air vehicles in both civil and military domains [6]. AC systems can provide service for ground networks, public safety, military communications, and improved cockpit data communications.
Data hungry high speed nodes with longer communication distances bring new research dimensions for the future communication systems in aeronautical domain. First of all, power efficiency and spectral efficiency are not usually equally important in aeronautical communication systems as in terrestrial communications. As the communication zones are larger in AC systems, in order to achieve longer distances, power is considered to be much more precious than bandwidth. Moreover, in aeronautical stations, power must be rigorously conserved since all of it is made on-board and must be shared among many power hungry systems [7].

It is important to note that, in order to achieve higher data rates to meet the requirements of improved avionic systems, it is desirable to transmit as much data as possible in the available bandwidth. Ideally, one would also like to use the smallest possible bandwidth to transmit the data, that is, by increasing the spectral efficiency. In the terrestrial, domain licenses for wireless bandwidth are becoming increasingly scarce and expensive. To the best of our knowledge, a bandwidth regulation in aeronautical domain is not as strict as terrestrial domain yet. However, using less bandwidth 


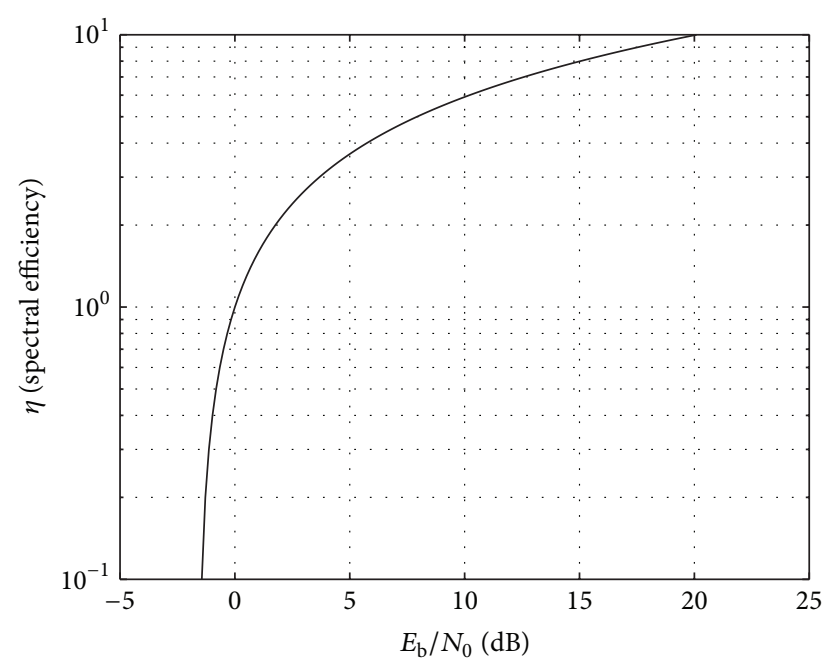

Figure 1: Capacity curve: limit of $\left(E_{\mathrm{b}} / N_{0}\right)_{\text {Req,Coded }}^{\mathrm{dB}}$.

reduces licensing costs and enables more nodes to share a given frequency band, therefore, always desirable. As opposed to above it is vital to note that in military/civil domain increasing the spectral efficiency is not desirable from an electronic warfare/robustness to interference point of view [8]. Moreover, reducing the spectral efficiency also enables longer distance communications by further reducing the required energy per bit according to Shannon (see Figure 1).

One of the most important issues on link budget analysis in AC domain is the antenna gains. Having a directed antenna with high gains reduces the delay/Doppler spread $[9,10]$, as well as reducing the transmit power of the AC system [11]. As the AC platform moves, the platform heading may shift (yaw) and the platform may tilt along the length (pitch), or from side to side (roll) [12]. Hence the antenna beamforming processing (mechanical or electronic) unit must rapidly compensate for the disturbances caused by the changes in yaw, pitch, and roll to maintain the link budget. This issue is related to the positions of the platforms, Euler angles of the platforms (yaw, pitch, and roll), and antenna gains [13].

Among all these (somewhat) contradicting requirements, analysis of an AC link budget becomes a critical importance. This paper analyze the AC systems from a link budget analysis point of view and define the requirements for the parameters of link budget with an emphasize on antenna gains. These requirements provide the basis for a robust AC link. We present a mathematical framework to provide an end-to-end link budget analysis utilizing the platform kinematics. Kinematics, link budget, and antenna gains are well investigated and understood in aeronautical [12], communications [14], and antenna engineering [8] domains separately; however, to the best of our knowledge a study of link budget emphasizing the effect of kinematics has not been done; that is, the link budget calculations generally assume static antenna gain [15]. The contribution of the mathematical framework provided in this study is twofold: (1) A real-time link budget analysis can be realized for AC system which depends on AC platforms positions and Euler angles. Given the telemetry data or flight plans of AC systems, this mathematical framework provides a run-time link budget analysis. This can be used to increase the performance of the AC systems in terms of data rates, power consumption, link margin, and so forth. (2) Mathematical framework of mapping the AC platform positions and Euler data to the platform antenna angles can be used for electronic beamforming and/or mechanical beam steering algorithms, which also provide a performance increase in AC systems in terms of link budget with increased gains and/or Doppler mitigation.

The rest of this paper is organized as follows. In Section 2, we present the system model for the analysis of link budget in AC systems and investigate the mathematical theory for the boundaries. In Section 3, we provide a tool to understand the kinematics of platforms and their effect on antenna gains/link margin as well as a mathematical background for the beamforming. In Section 4, the effectiveness of the tool is showed by numerical analysis, followed by the conclusions in Section 5.

\section{System Model for the Analysis of Link Budget in Aeronautical Communication Systems}

Received power at the input of the receiver chain in an aeronautical point-to-point communication link can be written as

$$
P_{\mathrm{r}}=\frac{P_{\mathrm{t}} G_{\mathrm{t}} G_{\mathrm{r}}}{L_{\text {backoff }} L_{\mathrm{tx}} L_{\mathrm{rx}} L_{\text {rain }} L_{\text {atm }} L_{\text {stat }} L_{\text {pol }} L_{\mathrm{fs}}},
$$

where $P_{\mathrm{t}}$ is maximum output power of power amplifier (PA), $G_{\mathrm{t}}$ is transmit antenna gain, $G_{\mathrm{r}}$ is receiver antenna gain, $L_{\text {backoff }}$ is back-off value, $L_{\text {tx }}$ is transmitter losses between PA and antenna, $L_{\mathrm{rx}}$ is receiver losses between antenna and low noise amplifier (LNA), $L_{\text {rain }}$ is rain losses, $L_{\text {atm }}$ is atmospheric losses, $L_{\text {stat }}$ is statistical losses due to multipath environment, $L_{\mathrm{pol}}$ is polarization losses, $L_{\mathrm{fs}}$ is free space path loss, $L_{\mathrm{fs}}=$ $(4 \pi d / \lambda)^{2}, \lambda$ is wavelength, $\lambda=c / f_{c}, c=3 \times 10^{8} \mathrm{~m} / \mathrm{s}, f_{c}$ is carrier frequency, and $d$ is distance between transmitter AC and receiver $\mathrm{AC}$.

Therefore, received energy per symbol over power spectral density of noise can be written as

$$
\left(\frac{E_{\mathrm{s}}}{N_{0}}\right)_{\text {Rec }}=\frac{P_{\mathrm{r}}}{N_{0} N_{\mathrm{F}} R_{\mathrm{s}} I_{\text {loss }}},
$$

where $E_{\mathrm{s}}$ is energy of symbol, $N_{0}$ is power spectral density of noise $-174 \mathrm{dBm} / \mathrm{Hz}, N_{0}=\kappa T^{0}, \kappa$ is Boltzmann constant $\kappa=1.38 \times 10^{23}, T^{0}$ is reference noise temperature (Kelvin), $N_{\mathrm{F}}$ is noise figure of the receiver $\mathrm{AC}, R_{\mathrm{s}}$ is symbol rate, $W$ is bandwidth, and $I_{\text {loss }}$ is implementation losses.

By using (1) and (2) one can find $\left(E_{s} / N_{0}\right)_{\mathrm{Rec}}^{\mathrm{dB}}=10 \times$ $\log _{10}\left(E_{\mathrm{s}} / N_{0}\right)_{\text {Rec }}$. Therefore calculating the link margin will be as follows:

$$
\mathrm{LM}^{\mathrm{dB}}=\left(\frac{E_{\mathrm{s}}}{N_{0}}\right)_{\mathrm{Rec}}^{\mathrm{dB}}-\left(\frac{E_{\mathrm{s}}}{N_{0}}\right)_{\text {Req }}^{\mathrm{dB}},
$$


where $\left(E_{\mathrm{s}} / N_{0}\right)_{\text {Req }}^{\mathrm{dB}}$ is the value corresponding to required energy of symbol over power spectral density of noise in order to ensure the bit error rate (BER) performance; say $P_{\mathrm{b}}$ :

$$
\left(\frac{E_{\mathrm{s}}}{N_{0}}\right)_{\text {Req }}^{\mathrm{dB}}=\left(\frac{E_{\mathrm{b}}}{N_{0}}\right)_{\text {Req,Coded }}^{\mathrm{dB}}+10 \times \log _{10} k,
$$

where $k$ is the information bit per symbol. The value of $k$ can be given as

$$
k=r m \text {, }
$$

where $r$ is the coding rate and $m=\log _{2} M, M$ is the modulation order. For a given modulation order and coding rate, one can find the value $k$ using (5). In order to calculate $\left(E_{\mathrm{s}} / N_{0}\right)_{\text {Req }}^{\mathrm{dB}}$ in (4) and $\mathrm{LM}^{\mathrm{dB}}$ in (3), we have to calculate $\left(E_{\mathrm{b}} / N_{0}\right)_{\text {Req,Coded }}^{\mathrm{dB}}$.

An important theoretical result from communication theory [16], called Shannon's Formula, gives the theoretical maximum data rate that can be reliably transmitted over an additive white Gaussian noise (AWGN) channel with bandwidth $(\mathrm{Hz})$, received power (watts), and noise PSD $N_{0}$ (watts/Hz):

$$
C=W \log _{2}\left(1+\frac{P_{\mathrm{r}}}{N_{0} W}\right) .
$$

Any reliable communication system must have a bit rate that is less than or equal to $C$, which is called the capacity of the channel. Spectral efficiency of a waveform is given by $\eta=$ $R_{\mathrm{b}} / W, R_{\mathrm{b}}=k R_{\mathrm{s}}$, where $k$ is given in (5), and power efficiency is measured by $E_{\mathrm{b}} / N_{0}$. Using these relationships and (6), we have

$$
\eta=\log _{2}\left(1+\frac{E_{\mathrm{b}}}{N_{0}} \eta\right)
$$

or

$$
\left(\frac{E_{\mathrm{b}}}{N_{0}}\right)_{\text {Req,Coded }}^{\mathrm{dB}} \geq \frac{2^{\eta}-1}{\eta} .
$$

In a wireless communication system, symbol rate $R_{\mathrm{s}}$ is calculated according to the digital-to-analog/analog-to-digital converter (DAC/ADC) speed (including oversampling) and $R_{\mathrm{b}}$ useful information bit rate. Bandwidth of the signal $W$ is generally given for a system due to regulations or in some cases might be restricted due to RF hardware. Anyhow, a given/selected $\eta=R_{\mathrm{b}} / W$ determines $\left(E_{\mathrm{b}} / N_{0}\right)_{\text {Req,Coded }}^{\mathrm{dB}}$.

Note that (8) provides a lower bound on for each value of $\left(E_{\mathrm{b}} / N_{0}\right)_{\text {Req,Coded }}^{\mathrm{dB}}$. However, this tradeoff between $\left(E_{\mathrm{b}} /\right.$ $\left.N_{0}\right)_{\text {Req, Coded }}^{\mathrm{dB}}$ and $\eta$ has a limit. No matter how much bandwidth you allow a signal to have, you can never use $\left(E_{\mathrm{b}} /\right.$ $\left.N_{0}\right)^{\mathrm{dB}}<-1.59 \mathrm{~dB}$ and still have reliable communication (see Figure 1).

Power and bandwidth are not usually equally important in aeronautical communication system. As the communication zones are larger in AC systems, in order to achieve longer distances power is considered to be much more precious than bandwidth. Moreover, in aeronautical stations, power must be rigorously conserved since all of it is made on-board and must be shared among many power hungry systems. Finally, it is important to note that increasing the bandwidth efficiency is not desirable from an interference mitigation point of view. Therefore from an aeronautical communication system design point of view, small $\left(E_{\mathrm{b}} / N_{0}\right)_{\text {Req,Coded }}^{\mathrm{dB}}$ is desirable (which means going left in Figure 1) in order to increase the LM as given in (3). On the other hand, for a given LM, lower $\left(E_{\mathrm{b}} / N_{0}\right)_{\mathrm{Req}, \text { Coded }}^{\mathrm{dB}}$ is desirable because it will let the system achieve the same BER performance with lower transmitting powers.

\section{Transmit/Receive Antenna Angles and Beamforming}

As discussed in the previous section, parameters that affect the AC system link budget can be summarized as follows: output power of PA, back-off value, transmit and receive antenna gains, transmitter/receiver side losses, rain losses, atmospheric losses, statistical losses due to multipath, polarization losses, carrier frequencies, communication distances, data rates, bandwidth, and noise figure. In order to provide a careful and robust system design all the above parameters as well as the relationship between them should be studied in detail. Even though most of these parameters are selected/dictated by the researchers/system requirements, integration related multidisciplinary subjects can be analyzed separately. In this section, we investigate the antenna gains according to the kinematics of platforms. In particular, we provide a mathematical tool for analyzing the transmitter and receiver antenna gains according to the positions of the platforms, Euler angles of the platforms (yaw, pitch, and roll), and antenna gain patterns.

The antenna gain pattern has a critical importance in system design. In AC systems, link margin can be increased (or the overall power consumption can be decreased) with a careful design of antenna pattern. A directivity can be placed by electronic beamforming and/or mechanical beam steering according to positions and Euler angles of the aeronautical platforms. This directivity will provide a higher transmitter and receiver antenna gain between platforms line-of-sight (LOS) and therefore can provide a robust AC system link budget with additional link margins. The relationship between increasing the transmitter and/or receiver antenna gain and link budget can be summarized as follows: increasing transmitter antenna gain $\left(G_{\mathrm{t}}\right)$ and/or receiver antenna gain $\left(G_{\mathrm{r}}\right)$, increase the received power $\left(P_{\mathrm{r}}\right)$ in (1), therefore also increasing the received energy per symbol over power spectral density of noise $\left(E_{\mathrm{s}} / N_{0}\right)_{\operatorname{Rec}}$ in (2). That means, careful design of antenna pattern utilizing the positions and Euler angles of the platforms yield increased $\left(E_{\mathrm{s}} / N_{0}\right)_{\mathrm{Rec}}$, which increase the link margin $\left(\mathrm{LM}^{\mathrm{dB}}\right)$ according to (3). Thus, tracking the positions and Euler angles of the platforms can increase the system performance from a link budget point of view. Moreover, it can provide transmission security and additional navigational reference to AC systems. 

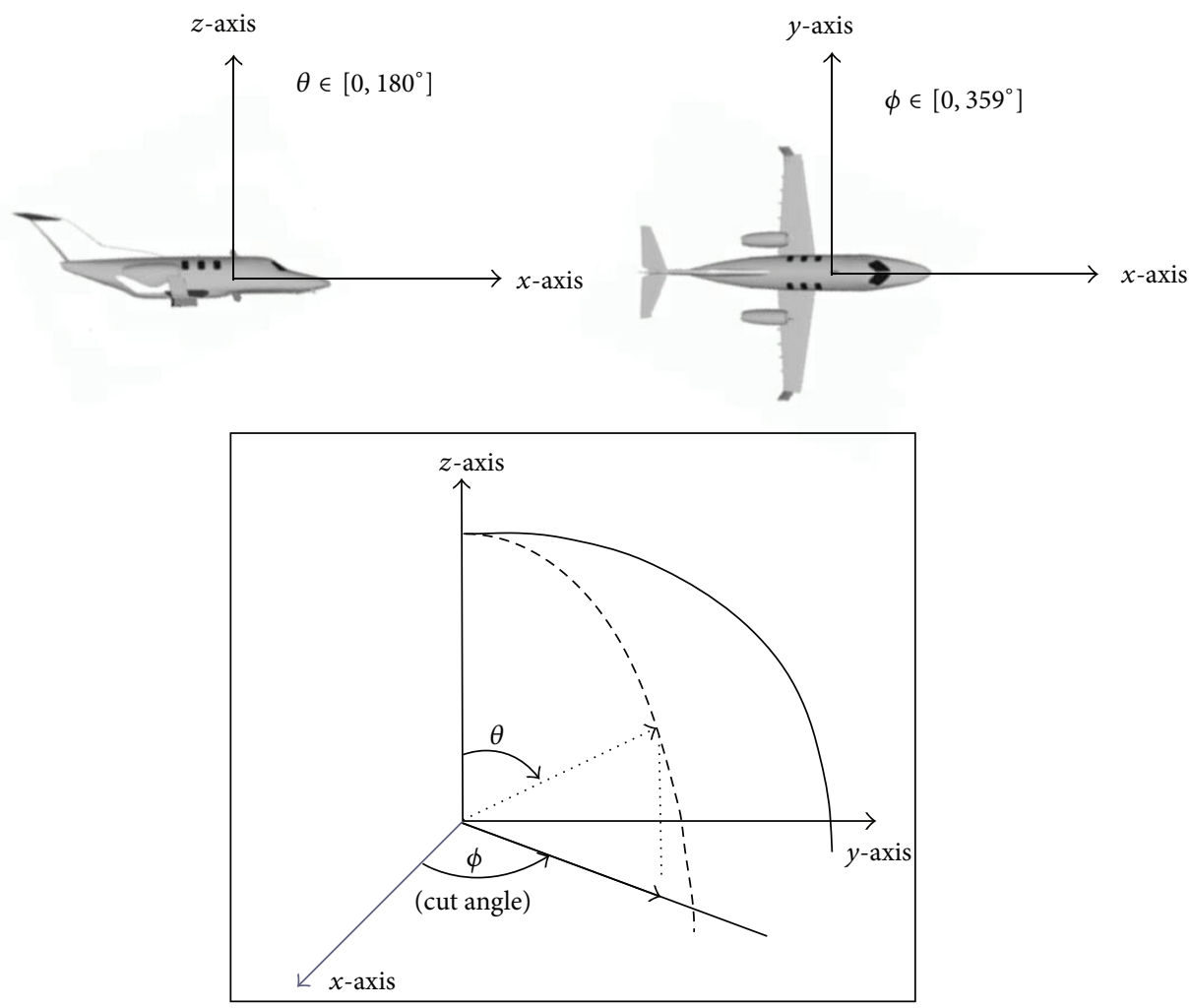

FIGURE 2: Antenna pattern angle definitions.

Although it is very well understood/studied in literature in different domains, a joint mathematical tool for both antenna engineers and aeronautical engineers could provide a complete understanding of system design. Therefore it is important to provide a basis to the antenna pattern angle definitions and aeronautical platform Euler angle definitions as in Figures 2 and 3, respectively.

Using the platform positions in Figures 4 and 5 and Eulerangle definitions, we study the LOS antenna angles for two platforms. (The rest of the paper will provide a formulation for two aeronautical platforms. However, the mathematical tools provided in this paper can be used for an aeronauticalground communication scenario s.t. a fixed platform with fixed Euler angles can be used for any of the platforms.) In this section, we first present the antenna angles for two platforms only based on positions. By using those results, we add the Euler angles to the formulations in order to provide a complete solution.

3.1. LOS Antenna Angles Based on Aeronautical Platform Positions. Let $\left(x_{1}, y_{1}, z_{1}\right)$ and $\left(x_{2}, y_{2}, z_{2}\right)$ denote the aeronautical platform 1 (AP1) and AP2 positions, respectively. Also consider that $\left(\right.$ pitch $_{1}$, roll $_{1}$, yaw $\left._{1}\right)$ and $\left(\right.$ pitch $_{2}$, roll $_{2}$, yaw $_{2}$ ) present the Euler angles for AP1 and AP2. The LOS antenna $\left(\theta_{i, 0,0,0}, i=1,2\right.$. $)$ angles for AP1 and AP2 in the absence of
Euler angles (i.e., $\left(\right.$ pitch $_{1}$, roll $_{1}$, yaw $\left.\left._{1}\right)=(0,0,0)\right)$ can be given as

$$
\begin{aligned}
& \theta_{1,0,0,0}= \begin{cases}\tan ^{-1}\left(\frac{\sqrt{\Delta x^{2}+\Delta y^{2}}}{\Delta z}\right), & \text { if } \Delta z \geq 0 \\
\pi+\tan ^{-1}\left(\frac{\sqrt{\Delta x^{2}+\Delta y^{2}}}{\Delta z}\right), & \text { if } \Delta z<0,\end{cases} \\
& \theta_{2,0,0,0}=\pi-\theta_{1,0,0,0},
\end{aligned}
$$

where $\Delta x=x_{2}-x_{1}, \Delta y=y_{2}-y_{1}$, and $\Delta z=z_{2}-z_{1}$. On the other hand, the LOS antenna $\left(\phi_{i, 0,0,0}, \quad i=1,2\right.$.) angles for AP1 and AP2 values can be calculated as

$$
\begin{aligned}
& \phi_{1,0,0,0}= \begin{cases}\tan ^{-1}\left(\frac{\Delta y}{\Delta x}\right), & \text { if } \Delta x \geq 0, \Delta y \geq 0 \\
\pi+\tan ^{-1}\left(\frac{\Delta y}{\Delta x}\right), & \text { if } \Delta x<0, \Delta y>0 \\
2 \pi+\tan ^{-1}\left(\frac{\Delta y}{\Delta x}\right), & \text { if } \Delta x>0, \Delta y<0 \\
\pi+\tan ^{-1}\left(\frac{\Delta y}{\Delta x}\right), & \text { if } \Delta x \leq 0, \Delta y \leq 0,\end{cases} \\
& \phi_{2,0,0,0}= \begin{cases}\phi_{1,0,0,0}+\pi, \quad \Delta y \geq 0 \\
\phi_{1,0,0,0}-\pi, \quad \Delta y<0 .\end{cases}
\end{aligned}
$$




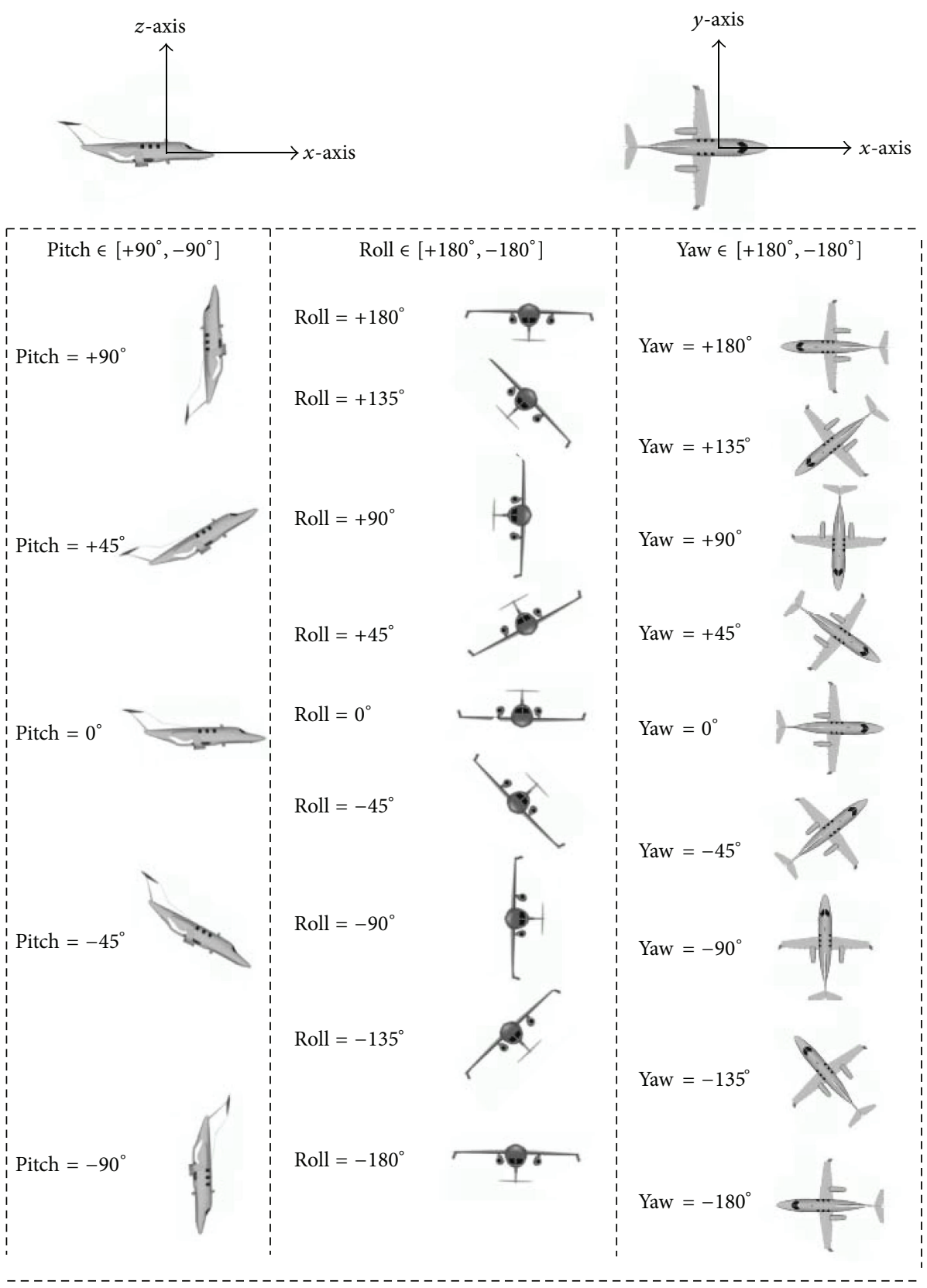

Figure 3: Aeronautical platform Euler angle definitions.

Note that, (9)-(10) provide the AP1 and the AP2 LOS angles in the absence of pitch, roll, and yaw angles. However, they will be used as the initial values for the rest of the paper.

3.2. LOS Antenna Angles Based on Aeronautical Platform Positions and Euler Angles. In this section, we first study the effect of yaw $_{i}, i=1,2$, then pitch $, i=1,2$, and finally roll $_{i}$, $i=1,2$, on the antenna angles. Firstly, we investigate the effect of platform yaw movement on the antenna angles. yaw $_{i}$ does not have any effect on $\theta_{i, 0,0,0}$; that is, $\theta_{i, \mathrm{yaw}_{i}, 0,0}=\theta_{i, 0,0,0}$. On the other hand, the effect of yaw ${ }_{i}$ on $\phi_{i, 0,0,0}$ can be given as

$$
\phi_{i, \text { yaw }_{i}, 0,0}= \begin{cases}\alpha_{i, \text { yaw }_{i}, 0,0}, & \text { if } 0 \leq \alpha_{i, \text { yaw }_{i}, 0,0}<2 \pi \\ \alpha_{i, \text { yaw }_{i}, 0,0}-2 \pi, & \text { if } 2 \pi \leq \alpha_{i, \text { aw }_{i}, 0,0} \\ \alpha_{i, \text { yaw }_{i}, 0,0}+2 \pi, & \text { if } \alpha_{i, \text { yaw }_{i} 0,0,0}<0,\end{cases}
$$

where $\alpha_{i, \mathrm{yaw}_{i}, 0,0}=\phi_{i, 0,0,0}+\mathrm{yaw}_{i}$.

After calculating $\theta_{i, \text { yaw }_{i}, 0,0}$ and $\phi_{i, \text { yaw }_{i}, 0,0}$ according to the yaw values of $\mathrm{AP} i, i=1,2$, we may calculate $\theta_{i, \mathrm{yaw}_{i}, \text {, } \text { itch }}, 0,0$ and 


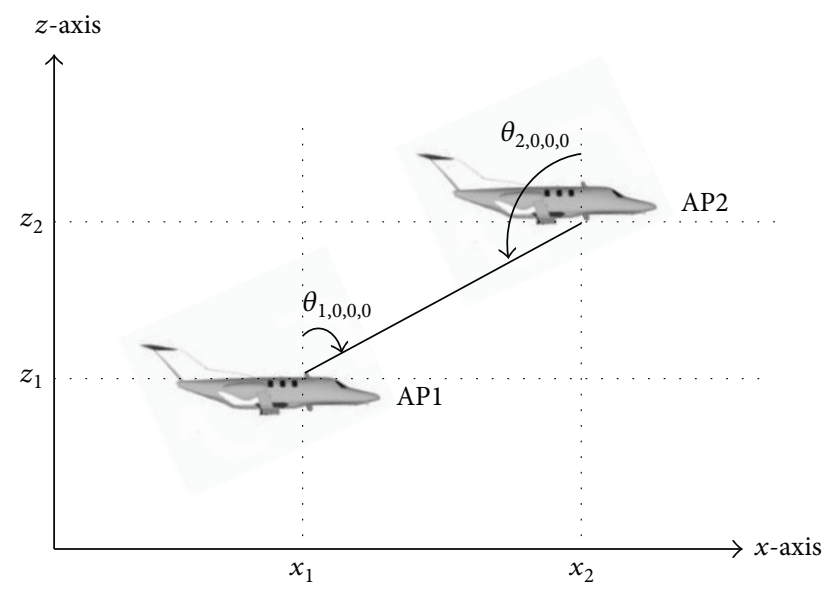

FIgURE 4: $\operatorname{LOS} \theta_{1,0,0,0}$ and $\theta_{2,0,0,0}$ angle definitions for AP1 and AP2.

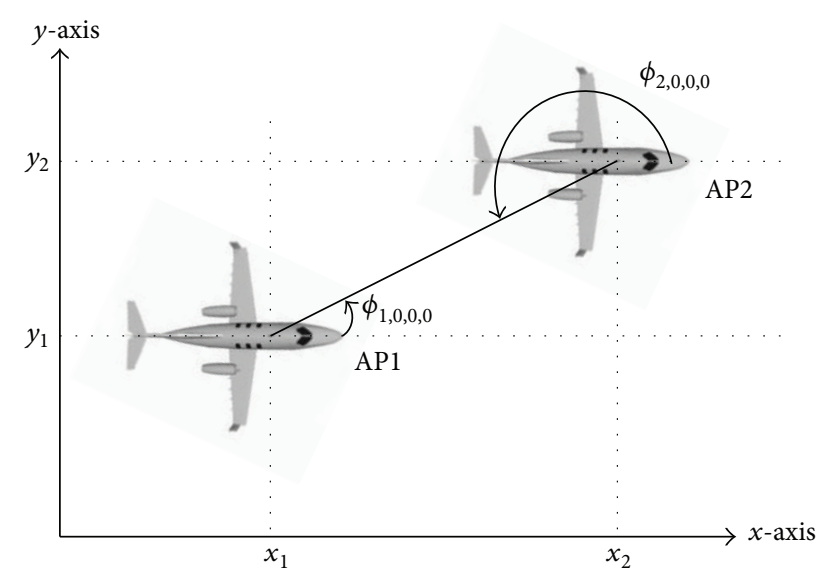

FIgURE 5: LOS $\phi_{1,0,0,0}$ and $\phi_{2,0,0,0}$ angle definitions for AP1 and AP2.

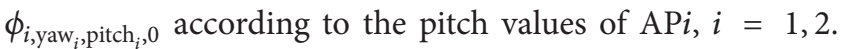
In order to present the effect of pitch on the angle, observe Figure 6. Let $\theta_{i, \mathrm{yaw}_{i}, 0,0}$ and $\phi_{i, \mathrm{yaw}_{i}, 0,0}, i=1,2$, be the angles calculated according to (11) which can be described with the point $A$ in Figure 6 . Then the platform's change of pitch angle $(\epsilon\{-\pi / 2, \pi / 2\})$ will result in the shift of $\theta_{i, \mathrm{yaw}_{i} \mathrm{pitch}_{i}, 0}$ and $\phi_{i, \text { yaw }_{i}, \text { pitch }_{i}, 0}$ on the circle which has the center of $O^{\prime}$.

Let $|A O|=R$; then $|A B|=R \cos \theta_{i, \text { yaw }_{i}, 0,0},|B O|=$ $R \sin \theta_{i, \text { yaw }_{i}, 0,0},\left|B O^{\prime}\right|=R \sin \theta_{i, \text { yaw }_{i}, 0,0} \cos \phi_{i, \mathrm{yaw}_{i}, 0,0},\left|O^{\prime} O\right|=$ $R \sin \theta_{i, \text { yaw }_{i}, 0,0} \sin \phi_{i, \text { yaw }_{i}, 0,0}$. Let the radius of the circle centered with $O^{\prime}$ be $\rho$. Since, $\rho^{2}+\left|O^{\prime} O\right|^{2}=R^{2}$, we can calculate $\rho=R \sqrt{1-\sin ^{2} \theta_{i, \text { yaw }_{i}, 0,0} \sin ^{2} \phi_{i, \text { yaw }_{i}, 0,0}}$. Let $\angle A O^{\prime} B$ be defined as $\gamma_{p, i}$, and then

$$
\begin{aligned}
\gamma_{p, i} & =\tan ^{-1}\left(\frac{|A B|}{\left|B O^{\prime}\right|}\right) \\
& =\tan ^{-1}\left(\frac{1}{\tan \theta_{i, \operatorname{yaw}_{i}, 0,0} \cos \phi_{i, \mathrm{yaw}_{i}, 0,0}}\right) .
\end{aligned}
$$

Therefore rewriting the $|A B|,|B O|$, and $\left|B O^{\prime}\right|$ lengths in terms of $\gamma_{p, i}$, we have

$$
\begin{aligned}
& |A B|=\rho \sin \gamma_{p, i}=R \sin \gamma_{p, i} \\
& \cdot \sqrt{1-\sin ^{2} \theta_{i, \mathrm{yaw}_{i}, 0,0} \sin ^{2} \phi_{i, \mathrm{yaw}_{i}, 0,0}}, \\
& \left|B O^{\prime}\right|=\rho \cos \gamma_{p, i}=R \cos \gamma_{p, i} \\
& \cdot \sqrt{1-\sin ^{2} \theta_{i, \mathrm{yaw}_{i}, 0,0} \sin ^{2} \phi_{i, \mathrm{yaw}_{i}, 0,0}} \\
& \left|O^{\prime} O\right|^{2}+\left|B O^{\prime}\right|^{2}=|B O|^{2}, \\
& |B O|=R\left[\cos ^{2} \gamma_{p, i}-\cos ^{2} \gamma_{p, i} \sin ^{2} \theta_{i, \mathrm{yaw}_{i}, 0,0} \sin ^{2} \phi_{i, \mathrm{yaw}_{i}, 0,0}\right. \\
& \left.\quad+\sin ^{2} \theta_{i, \mathrm{yaw}_{i}, 0,0} \sin ^{2} \phi_{i, \mathrm{yaw}_{i}, 0,0}\right]^{1 / 2} .
\end{aligned}
$$

When we have a tilt along the length in the platform (i.e., a change of pitch ${ }_{i}$ ), we will have a new point on the circle centered with $O^{\prime}$. Let this point be $A^{\prime}$ and the projection of this point on $x-y$ plane be $B^{\prime}$. Then we can calculate the angle $\angle A^{\prime} O^{\prime} B^{\prime}=\gamma_{p, i}-$ pitch $_{i}$. Using this relationship and (13) we may write $\left|A^{\prime} B^{\prime}\right|,\left|B^{\prime} O\right|$, and $\left|B^{\prime} O^{\prime}\right|$ as follows:

$$
\begin{aligned}
& \left|A^{\prime} B^{\prime}\right|=R \sin \left(\gamma_{p, i}-\operatorname{pitch}_{i}\right) \sqrt{1-\sin ^{2} \theta_{i, \mathrm{yaw}_{i}, 0,0} \sin ^{2} \phi_{i, \mathrm{yaw}_{i}, 0,0}} \\
& \left|B^{\prime} O^{\prime}\right|=R \cos \left(\gamma_{p, i}-\operatorname{pitch}_{i}\right) \sqrt{1-\sin ^{2} \theta_{i, \mathrm{yaw}_{i}, 0,0} \sin ^{2} \phi_{i, \mathrm{yaw}_{i}, 0,0}}, \\
& \left|B^{\prime} O\right|=R\left[\cos ^{2}\left(\gamma_{p, i}-\operatorname{pitch}_{i}\right)-\cos ^{2}\left(\gamma_{p, i}-\operatorname{pitch}_{i}\right) \sin ^{2} \theta_{i, \mathrm{yaw}_{i}, 0,0} \sin ^{2} \phi_{i, \mathrm{yaw}_{i}, 0,0}+\sin ^{2} \theta_{i, \mathrm{yaw}_{i}, 0,0} \sin ^{2} \phi_{i, \mathrm{yaw}_{i}, 0,0}\right]^{1 / 2}, \\
& \alpha_{i, \mathrm{yaw}_{i}, \mathrm{pitch}_{i}, 0} \\
& =\tan ^{-1}\left(\frac{\sqrt{\cos ^{2}\left(\gamma_{p, i}-\operatorname{pitch}_{i}\right)-\cos ^{2}\left(\gamma_{p, i}-\operatorname{pitch}_{i}\right) \sin ^{2} \theta_{i, \text { yaw }_{i}, 0,0} \sin ^{2} \phi_{i, \text { yaw }_{i}, 0,0}+\sin ^{2} \theta_{i, \text { aaw }_{i}, 0,0} \sin ^{2} \phi_{i, \text { yaw }_{i}, 0,0}}}{\sqrt{1-\sin ^{2} \theta_{i, \mathrm{yaw}_{i}, 0,0} \sin ^{2} \phi_{i, \mathrm{yaw}_{i}, 0,0}}}\right), \\
& \beta_{i, \mathrm{yaw}_{i} \mathrm{pitch}_{i}, 0}=\tan ^{-1}\left(\frac{\sin \theta_{i, \mathrm{yaw}_{i}, 0,0} \sin \phi_{i, \mathrm{yaw}_{i}, 0,0}}{\sqrt{1-\sin ^{2} \theta_{i, \mathrm{yaw}_{i}, 0,0} \sin ^{2} \phi_{i, \mathrm{yaw}_{i}, 0,0}} \cos \left(\gamma_{p, i}-\mathrm{pitch}_{i}\right)}\right) .
\end{aligned}
$$


Let $\alpha_{i, \text { yaw }_{i}, \text { pitch }_{i}, 0}=\tan ^{-1}\left(\left|B^{\prime} O\right| /\left|A^{\prime} B^{\prime}\right|\right)$ and $\beta_{i, \text { yaw }_{i}, \text { pitch }_{i}, 0}=$ $\tan ^{-1}\left(\left|O^{\prime} O\right| /\left|B^{\prime} O^{\prime}\right|\right)$ be the angles calculated as (17) and (18), respectively. Then according to the pitch value $\left(\mathrm{pitch}_{i}\right)$ of $\mathrm{AP} i$, $i=1,2, \theta_{i, \text { yaw }_{i}, \text { pitch }_{i}, 0}$ and $\phi_{i, \text { yaw }_{i}, \text { pitch }_{i}, 0}$ can be calculated as

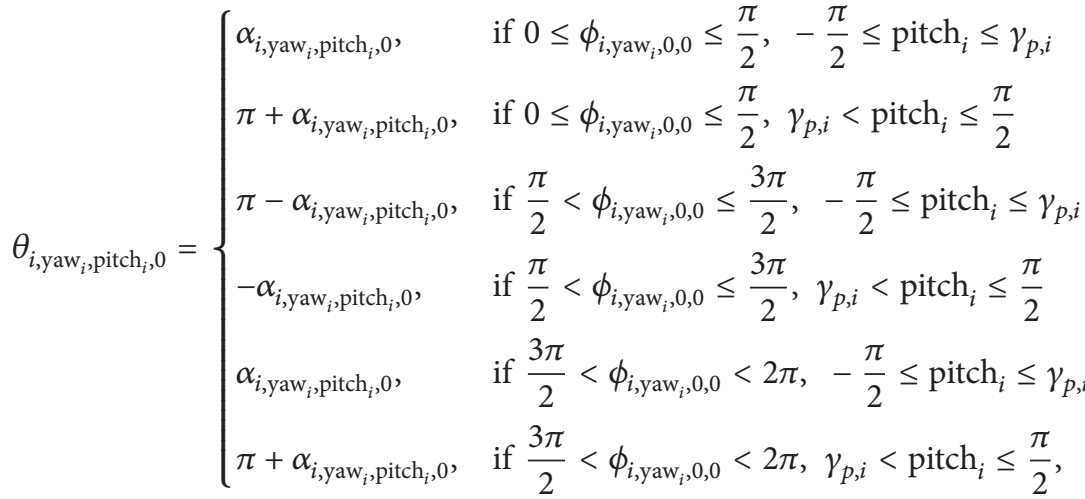

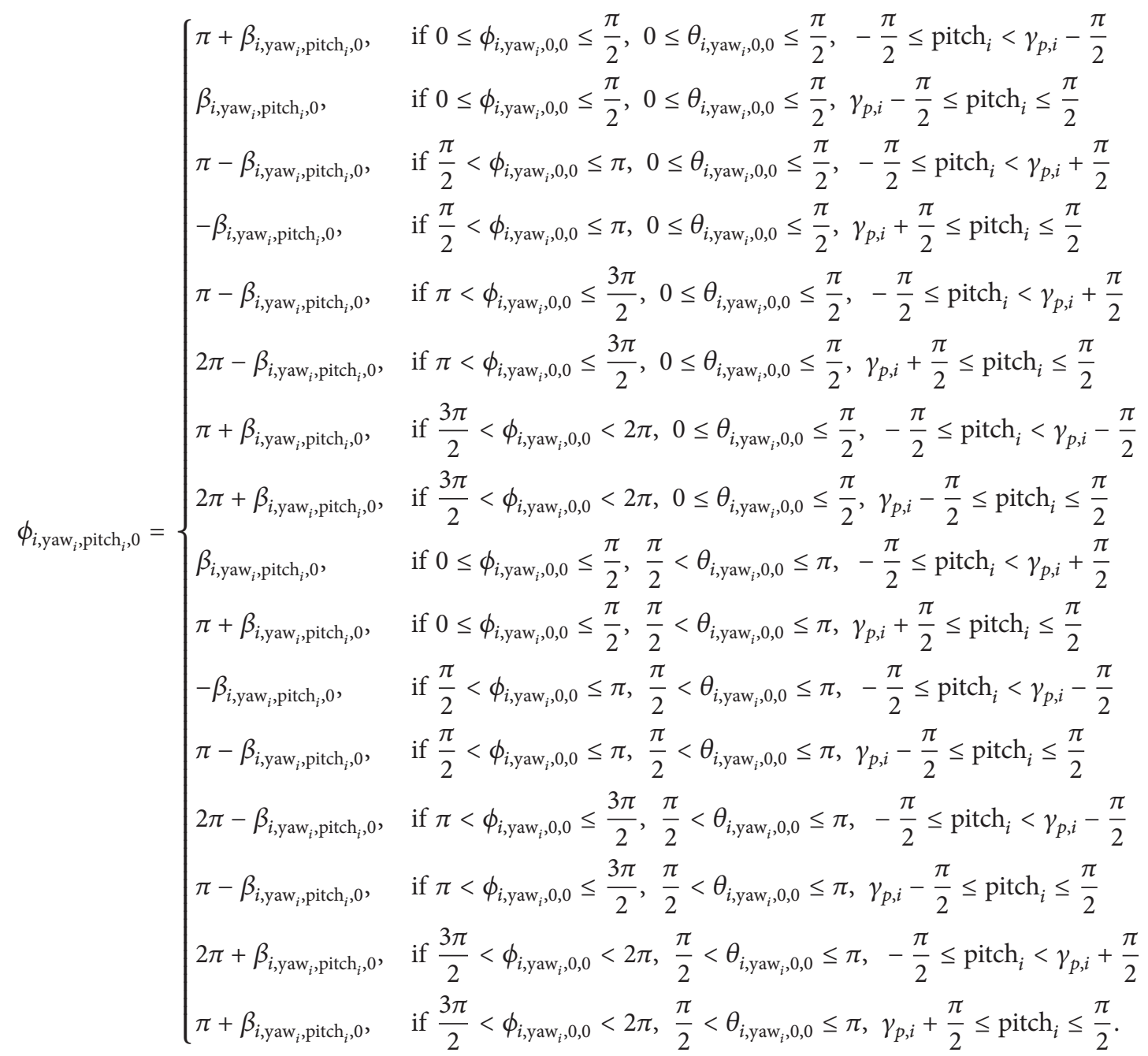

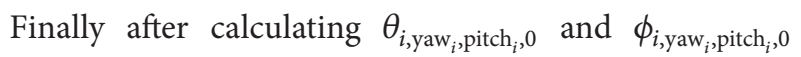
according to the pitch and yaw values of $\mathrm{AP} i, i=1,2$, we may calculate $\theta_{i, \text { yaw }_{i}, \text { pitch }_{i}, \text { roll }_{i}}$ and $\phi_{i, \text { yaw }_{i}, \text { pitch }_{i}, \text { roll }_{i}}$ according to the roll values of $\mathrm{AP} i, i=1,2$, in a similar manner.

Figure 7 presents the effect of roll on the antenna angles. Let $\theta_{i, \text { yaw }_{i}, \text { pitch }_{i}, 0}$ and $\phi_{i, \text { yaw }_{i}, \text { pitch }_{i}, 0}, i=1,2$, be the angles calculated according to (19) which can be described with the point $A$. Then the platform's change of roll angle $(\in\{-\pi, \pi\})$

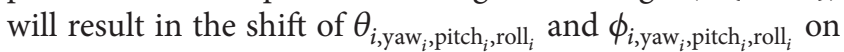
the circle which has the center of $O^{\prime}$.

Let $|A O|=R$; then $|A B|=R \cos \theta_{i, \text {,aw }_{i} \text {, } \text { pitch }_{i}, 0},|B O|=$ $R \sin \theta_{i, \text { yaw }_{i}, \text { pitch }_{i}, 0},\left|B O^{\prime}\right|=R \sin \theta_{i, \text {,aw }_{i}, \text { pitch }_{i}, 0} \sin \phi_{i, \text { yaw }_{i}, \text { pitch }_{i}, 0}$, 


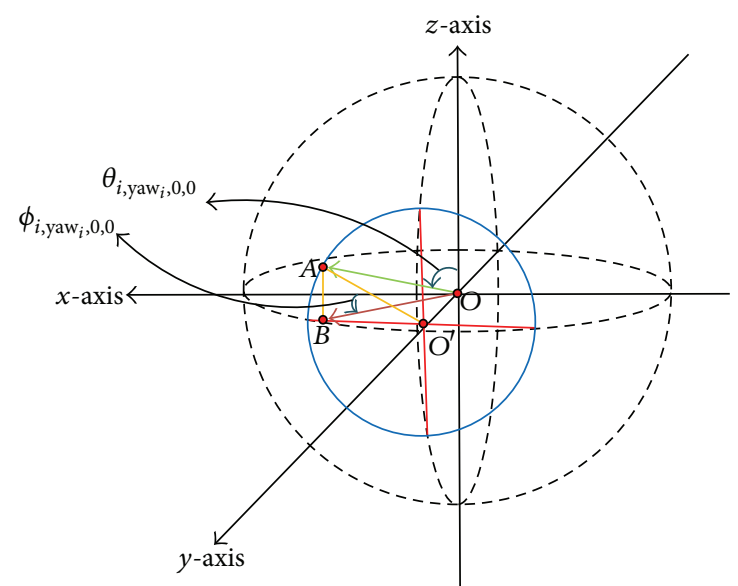

FigurE 6: Effect of platform pitch angle to the antenna angles.
$\left|O^{\prime} O\right|=R \sin \theta_{i, \text { yaw }_{i}, \text { pitch }_{i}, 0} \cos \phi_{i, \text { yaw }_{i}, \text { pitch }_{i}, 0}$. Let the radius of the circle centered with $O^{\prime}$ be $\rho$. Since, $\rho^{2}+\left|O^{\prime} O\right|^{2}=R^{2}$, we can calculate $\rho=R \sqrt{1-\sin ^{2} \theta_{i, \text { yaw }_{i}, \text { pitch }_{i}, 0} \cos ^{2} \phi_{i, \text { yaw }_{i}, \text { pitch }_{i}, 0}}$. Let $\angle A O^{\prime} B$ be $\left(\gamma_{r, i}\right)$, and then

$$
\begin{aligned}
\gamma_{r, i} & =\tan ^{-1}\left(\frac{|A B|}{\left|B O^{\prime}\right|}\right) \\
& =\tan ^{-1}\left(\frac{1}{\tan \theta_{i, \text { yaw }_{i}, \text { pitch }_{i}, 0} \sin \phi_{i, \text { yaw }_{i}, \text { pitch }_{i}, 0}}\right) .
\end{aligned}
$$

When we have tilt from side to side in the platform (i.e., a change of roll $_{j}$ ), we will have a new point on the circle centered with $O^{\prime}$. Let this point be $A^{\prime}$ and the projection of this point on $x-y$ plane be $B^{\prime}$. Then we can calculate the angle $\angle A^{\prime} O^{\prime} B^{\prime}=\gamma_{r, i}-$ roll $_{i}$. Similar calculations as given for pitch, we may write $\left|A^{\prime} B^{\prime}\right|,\left|B^{\prime} O\right|$, and $\left|B^{\prime} O^{\prime}\right|$ as

$$
\begin{aligned}
& \left|A^{\prime} B^{\prime}\right|=R \sqrt{1-\sin ^{2} \theta_{i, \mathrm{yaw}_{i}, \mathrm{pitch}_{i}, 0} \cos ^{2} \phi_{i, \mathrm{yaw}_{i}, \mathrm{pitch}_{i}, 0}} \cdot \sin \left(\gamma_{r, i}-\operatorname{roll}_{i}\right), \\
& \left|B^{\prime} O^{\prime}\right|=R \sqrt{1-\sin ^{2} \theta_{i, \mathrm{yaw}_{i}, \mathrm{pitch}_{i}, 0} \cos ^{2} \phi_{i, \mathrm{yaw}_{i}, \mathrm{pitch}_{i}, 0}} \cdot \cos \left(\gamma_{r, i}-\operatorname{roll}_{i}\right), \\
& \left|B^{\prime} O\right|=R\left(\cos ^{2}\left(\gamma_{r, i}-\operatorname{roll}_{i}\right)-\cos ^{2}\left(\gamma_{r, i}-\operatorname{roll}_{i}\right) \sin ^{2} \theta_{i, \mathrm{yaw}_{i}, \mathrm{pitch}_{i}, 0} \cos ^{2} \phi_{i, \mathrm{yaw}_{i}, \mathrm{pitch}_{i}, 0}+\sin ^{2} \theta_{i, \mathrm{yaw}_{i}, \mathrm{pitch}_{i}, 0} \cos ^{2} \phi_{i, \mathrm{yaw}_{i}, \mathrm{pitch}_{i}, 0}\right)^{1 / 2},
\end{aligned}
$$

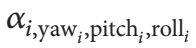

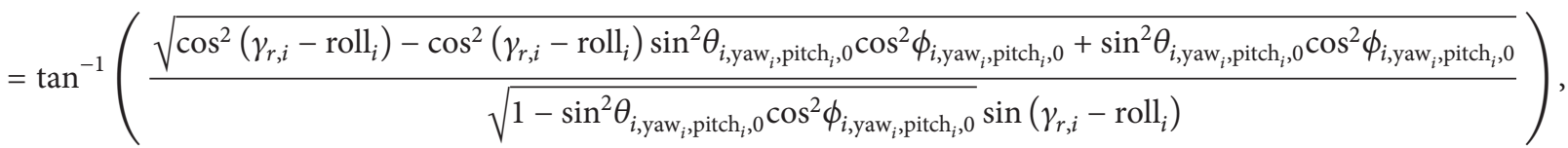

$$
\begin{aligned}
& \beta_{i, \text { yaw }_{i}, \text { pitch }_{i}, \text { roll }_{i}}=\tan ^{-1}\left(\frac{\sqrt{1-\sin ^{2} \theta_{i, \text { yaw }_{i}, \text { pitch }_{i}, 0} \cos ^{2} \phi_{i, \mathrm{yaw}_{i}, \text { pitch }_{i}, 0}} \cos \left(\gamma_{r, i}-\operatorname{roll}_{i}\right)}{\sin \theta_{i, \mathrm{yaw}_{i}, \mathrm{pitch}_{i}, 0} \cos \phi_{i, \mathrm{yaw}_{i}, \mathrm{pitch}_{i}, 0}}\right) \text {. }
\end{aligned}
$$

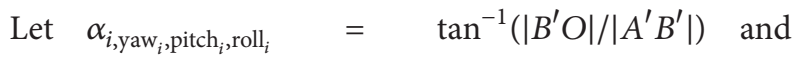
$\beta_{i, \text { yaw }_{i}, \text {,itch }_{i}, \text { roll }_{i}}=\tan ^{-1}\left(\left|B^{\prime} O^{\prime}\right| /\left|O^{\prime} O\right|\right)$ be the angles calculated as (24) and (25), respectively. Then according to the roll value

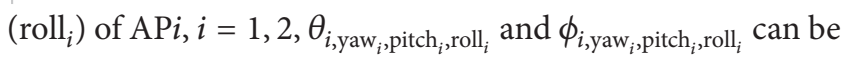
calculated as in

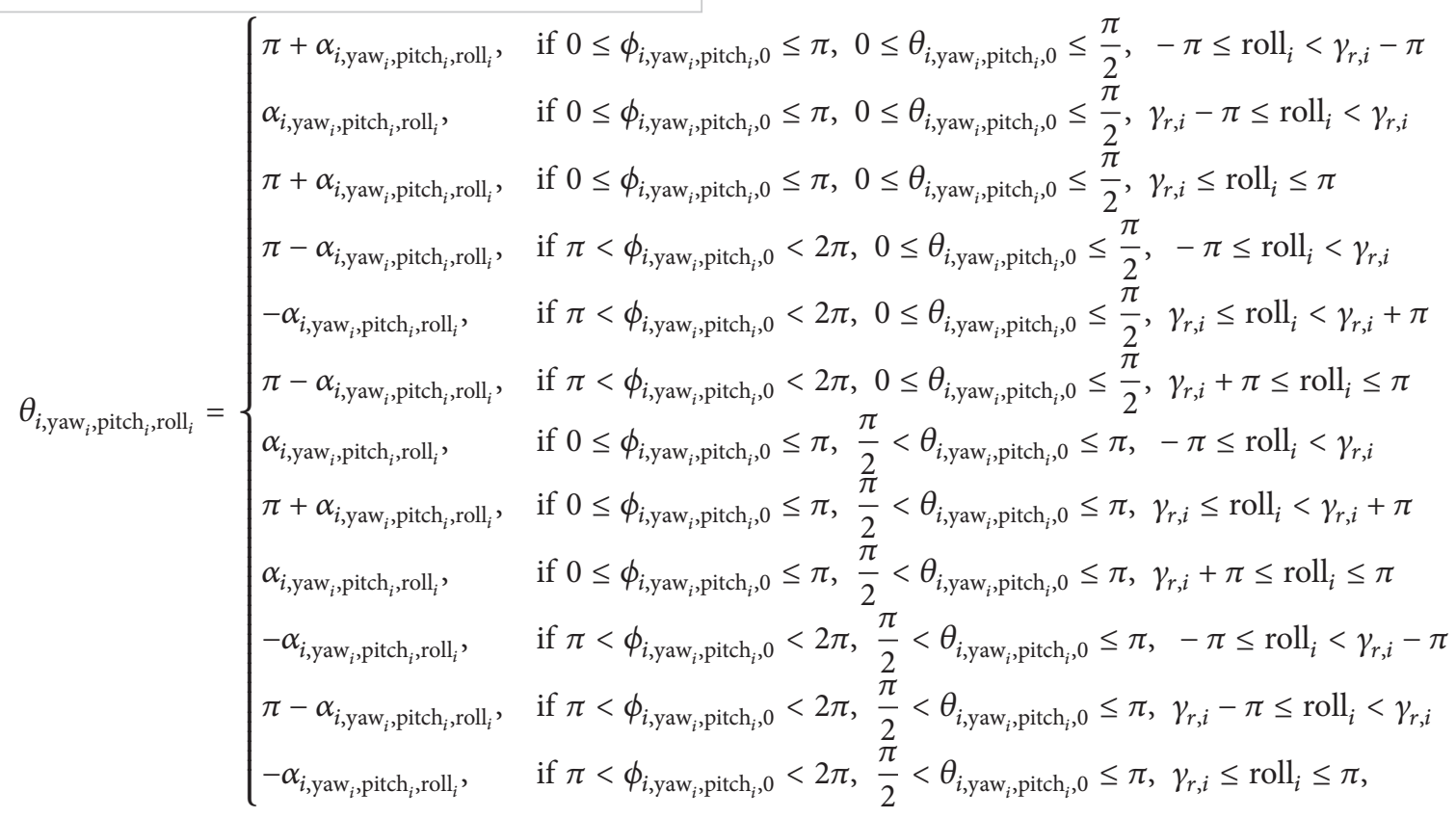




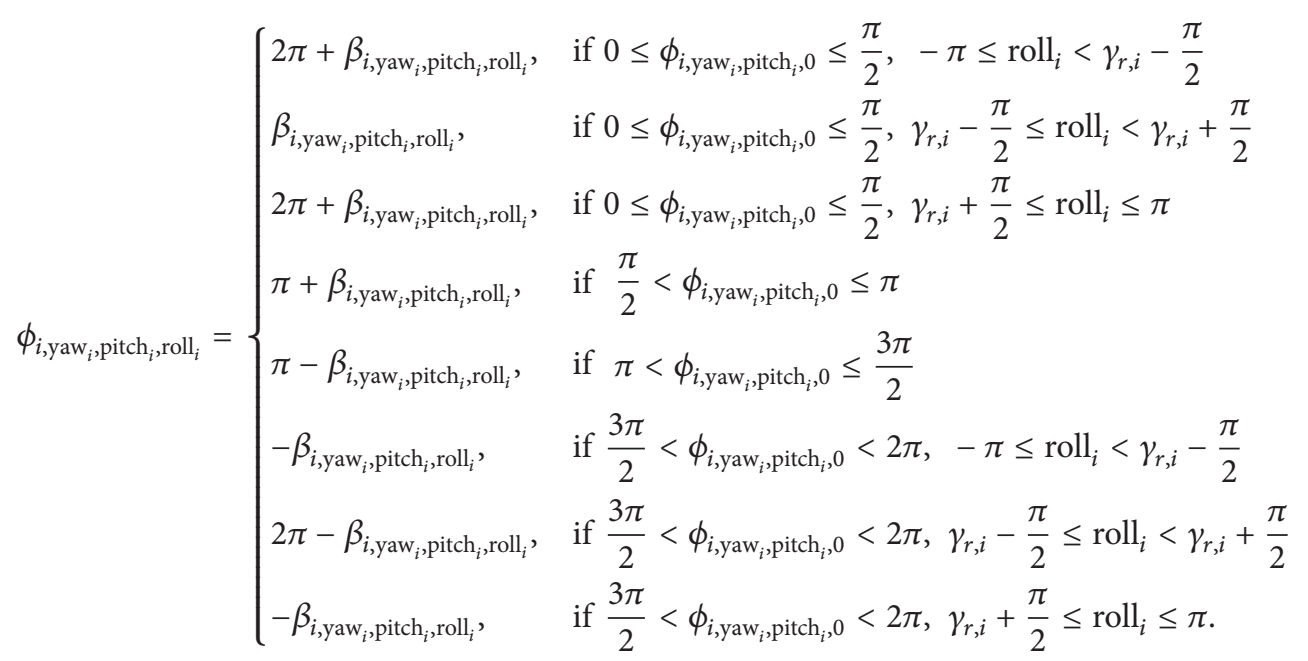

\section{Numerical Analysis}

In this section, we present numerical analysis based on typical AC scenarios that covers easy-to-understand positions

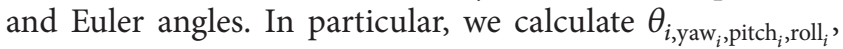
$\phi_{i, \text { yaw }_{i}, \text {,itch }_{i}, \text { roll }_{i}}$ using $\left\{\theta_{i, 0,0,0}, \phi_{i, 0,0,0}\right\},\left\{\theta_{i, \text { yaw }_{i}, 0,0}, \phi_{i, \text { yaw }_{i}, 0,0}\right\}$, and $\left\{\theta_{i, \text { yaw }_{i}, \text { pitch }_{i}, 0}, \phi_{i, \text { yaw }_{i}, \text { pitch }_{i}, 0}\right\}$. Table 1 presents the results of the numerical analysis. (In this study, we use practical measurements and our tool had a chance to be used in a real-time practical scenario where the position and Euler angles are collected by the telemetry of the aeronautical stations. The verification and validation activities are done using the practical scenarios; however, as the telemetry has a limited continuous data (i.e., relative positions and/or Euler angles are slowly changing within boundaries due to physical limitations of the ASs), the results do not provide enough statistics to verify all conditions. Therefore, we present a set of snapshot scenarios that cover many possible environments and without sake of brevity; we continue our analytical analysis which leads to results that can be easily followed with geometrical verification as provided in Table 1.) Note that, $\theta_{i, \text { yaw }_{i}, \text { pitch }_{i}, \text {,oll }_{i}}, \phi_{i, \text { yaw }_{i}, \text { pitch }_{i}, \text {,oll }_{i}}$ values are calculated with a precision of $2^{\circ}$, that is, according to the antenna gain value outputs of Computer Simulation Technology (CST) Microwave Studio Suite to decrease the processing power. Depending on the link budget analysis, one may allow having antenna pointing error which can be covered by link margin to provide error tolerant signal processing algorithms for the antenna beamforming. (It is important to note that one can easily extend the study for real-time link budget calculations and beamforming study, utilizing positions and Euler angles of platforms with a time index for a real scenario.)

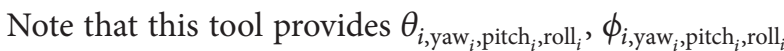
values using the position and Euler angles of the plat-

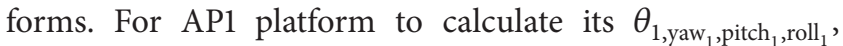

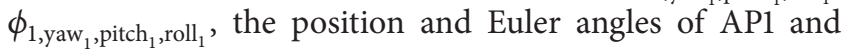
position of AP2 will be enough. In most of the avionics usage scenario, this assumption is reasonable as the platforms know each other's initial positions via surveillance systems, additional avionic systems that provide position values with very low data rate communication or RADAR; however they do not know each other's Euler angles. Using this mathematical tool and mechanical/electronical beam steering algorithms, the positions of the aircraft can be followed, while a robust communication can be ensured with increased link margins with less power burden on the platform.

\section{Conclusion}

In this paper, we investigate the link budget of an AC system and provide a tool for antenna beamforming. In particular, we present the relationship between link budget analysis and antenna gains. Then, using the position information and Euler angles of the platforms, we provide a tool that calculates the antenna angles. This tool enables real-time link budget analysis of AC and Doppler/delay spread mitigation techniques to perform better, as well as providing a complete set of mathematical tool for a beam steering (electrical/mechanical) algorithm.

\section{Competing Interests}

The authors declare that there is no conflict of interests regarding the publication of this paper.

\section{Acknowledgments}

The authors gratefully acknowledge the contributions of their colleague Ali Ozgur Tasoglu for his work on the original version of this document. M. Cenk Erturk, faculty advisor of Yasin Aksan, also would like to thank his student on implementations of the tool in this paper. 


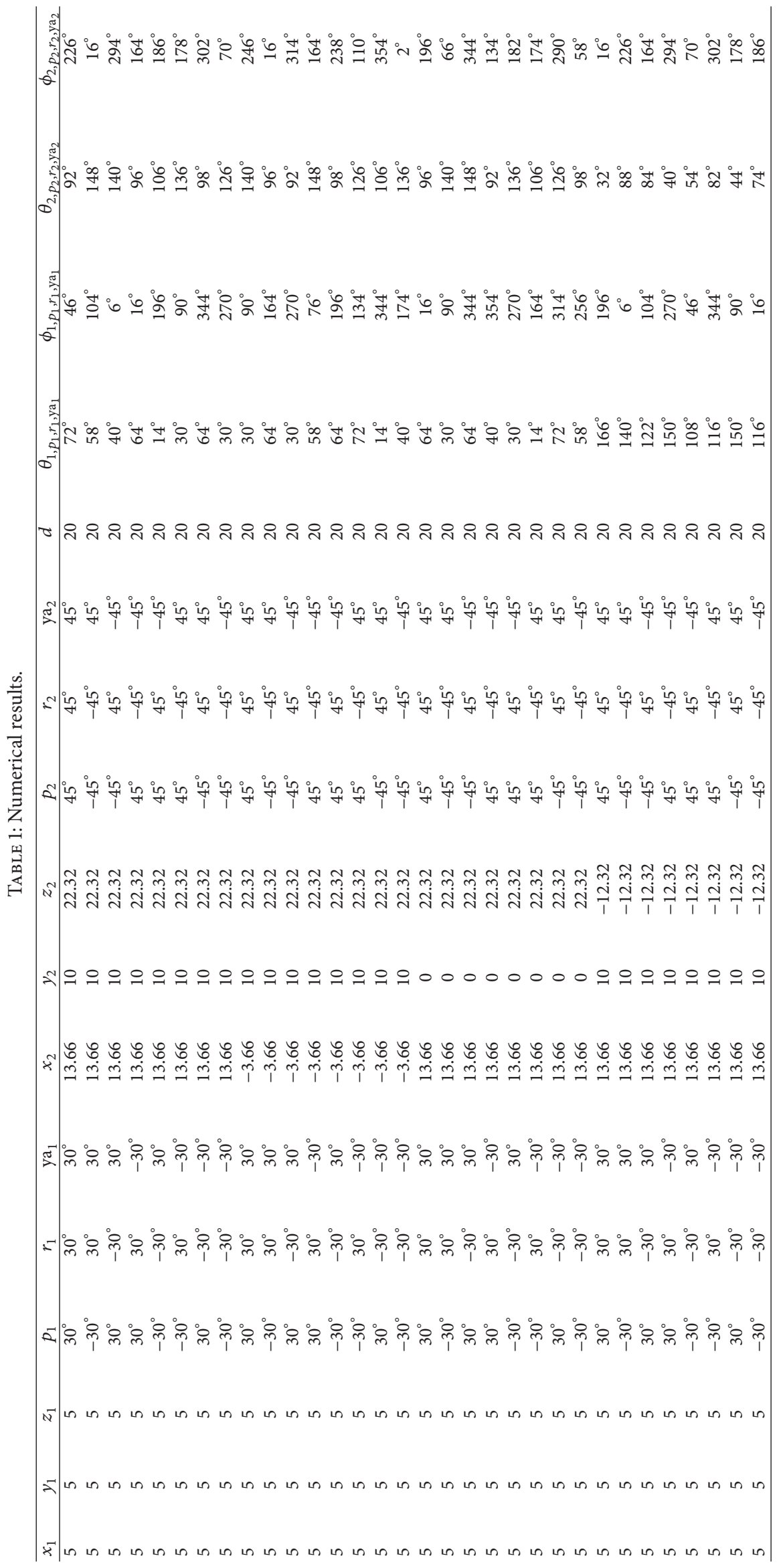




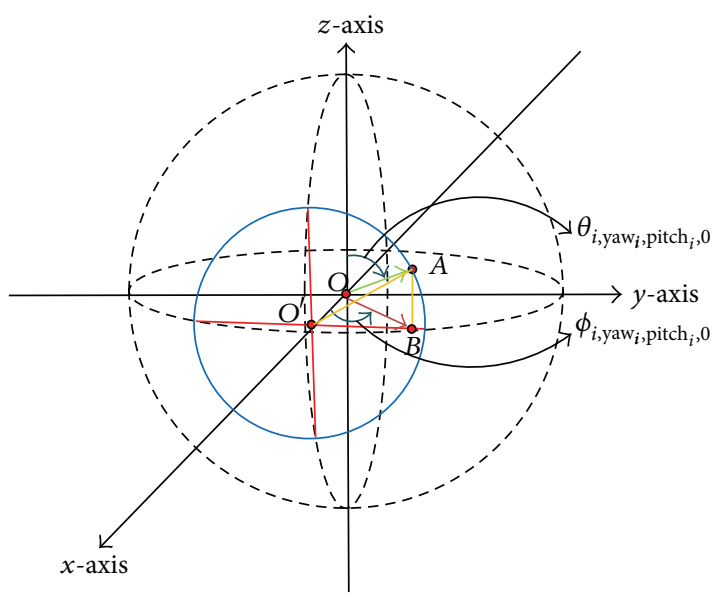

FIGURE 7: Effect of platform roll angle to the antenna angles.
[13] C. L. Yu, W. D. Burnside, and M. C. Gilreath, "Volumetric pattern analysis of airborne antennas," IEEE Transactions on Antennas and Propagation, vol. 26, no. 5, pp. 636-641, 1978.

[14] D. Stacey, Aeronautical Radio Communication Systems and Networks, John Wiley \& Sons, England, UK, 2008.

[15] International Civil Aviation Organization (ICAO), "Comparison of typical air/ground aeronautical communication system propagation losses in the $\mathrm{L}$ band and the $\mathrm{C}$ band," in Proceedings of the Regional Preparatory Group Meeting for World Radiocommunication Conference (WRC '05), Bangkok, Thailand, February 2005.

[16] J. G. Proakis, Digital Communications, McGraw-Hill, New York, NY, USA, 1995.

\section{References}

[1] M. Schnell and S. Scalise, "NEWSKY_concept for NEtworking the SKY for civil aeronautical communications," IEEE Aerospace and Electronic Systems Magazine, vol. 22, no. 5, pp. 25-29, 2007.

[2] NASA-ACAST, "Future aeronautical communication infrastructure technology investigation," 2008, http://acast.grc .nasa.gov/main/projects/.

[3] R. J. Kerczewski, "Aeronautical communications research and development needs for future air traffic management applications," in Proceedings of the IEEE Aerospace Conference, pp. 1169-1176, Big Sky, MT, USA, March 2002.

[4] E. Sakhaee and A. Jamalipour, "The global in-flight internet," IEEE Journal on Selected Areas in Communications, vol. 24, no. 9, pp. 1748-1757, 2006.

[5] J. Lai, "Broadband wireless communication systems provided by commercial airplanes," US Patents 6285 878, 2001.

[6] J. M. I. Alonso and M. S. Pérez, "Phased array for UAV communications at $5.5 \mathrm{GHz}$," IEEE Antennas and Wireless Propagation Letters, vol. 14, pp. 771-774, 2014.

[7] J. Chen and T. G. Pratt, "Transmit energy-efficiency for long-range wireless communications from battery-powered unmanned systems," IEEE Transactions on Aerospace and Electronic Systems, vol. 51, no. 4, pp. 2944-2959, 2015.

[8] G. M. Djuknic, J. Freidenfelds, and Y. Okunev, "Establishing wireless communications services via high-altitude aeronautical platforms: a concept whose time has come?" IEEE Communications Magazine, vol. 35, no. 9, pp. 128-135, 1997.

[9] M. C. Erturk, J. Haque, W. A. Moreno, and H. Arslan, "Doppler mitigation in OFDM-based aeronautical communications," IEEE Transactions on Aerospace and Electronic Systems, vol. 50, no. 1, pp. 120-129, 2014.

[10] S. L. Heath, G. L. McAninch, C. D. Smith, and D. A. Conner, "Validation of ray tracing code refraction effect," in Proceedings of the AIAA/CEAS Aeroacoustics Conference, pp. 1-11, May 2002.

[11] T. J. Willink, C. C. Squires, G. W. Colman, and M. T. Muccio, "Measurement and characterization of low-altitude airto-ground MIMO channels," IEEE Transactions on Vehicular Technology, vol. 65, no. 4, pp. 2637-2648, 2016.

[12] J. Farrell and M. Barth, The Global Positioning System and Inertial Navigation, McGraw-Hill, New York, NY, USA, 1998. 


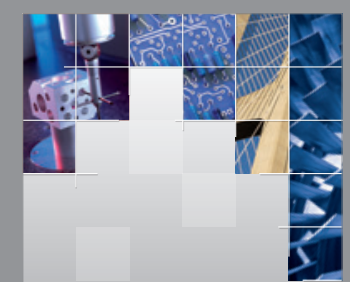

\section{Enfincering}
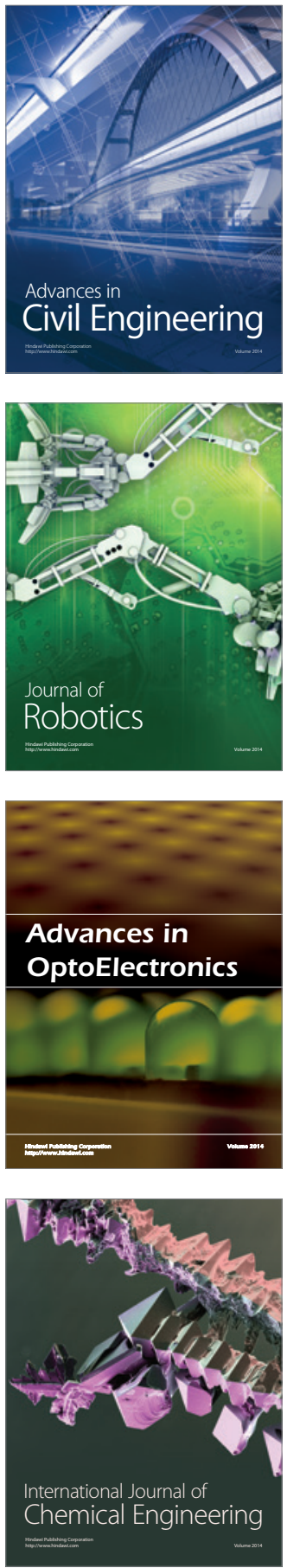

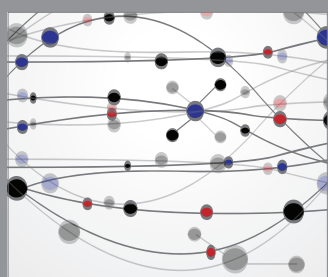

The Scientific World Journal

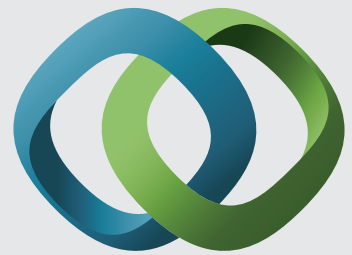

\section{Hindawi}

Submit your manuscripts at

http://www.hindawi.com
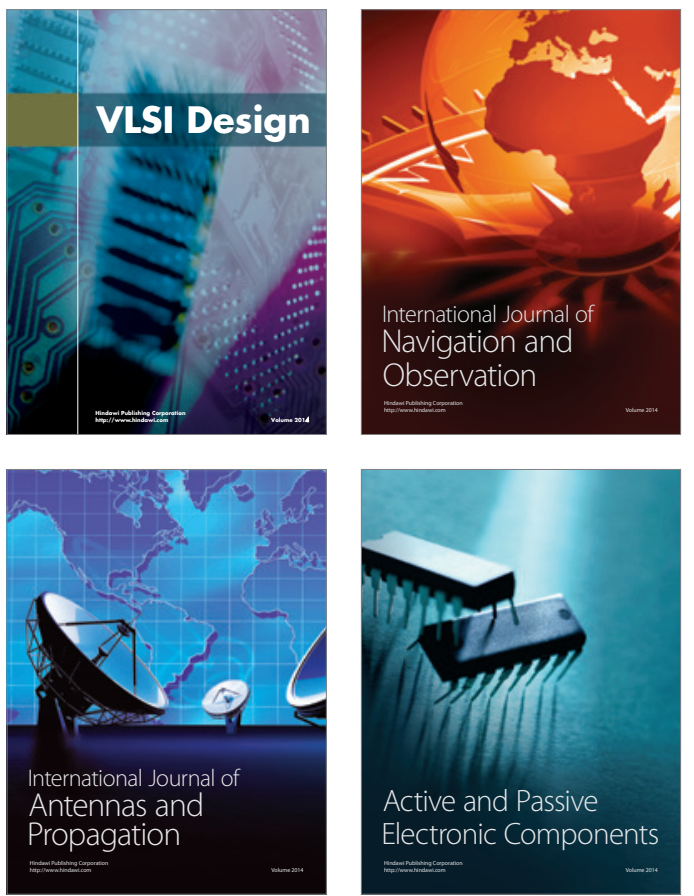
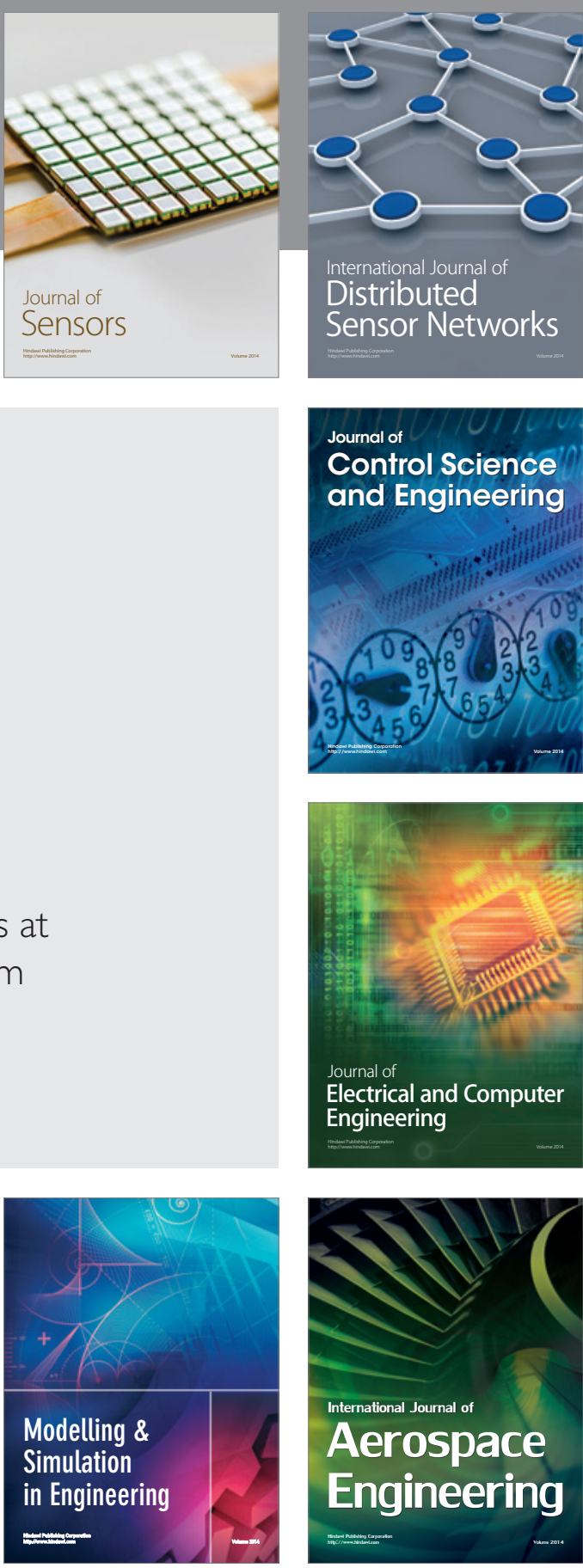

International Journal of

Distributed

Sensor Networks

Journal of

Control Science

and Engineering
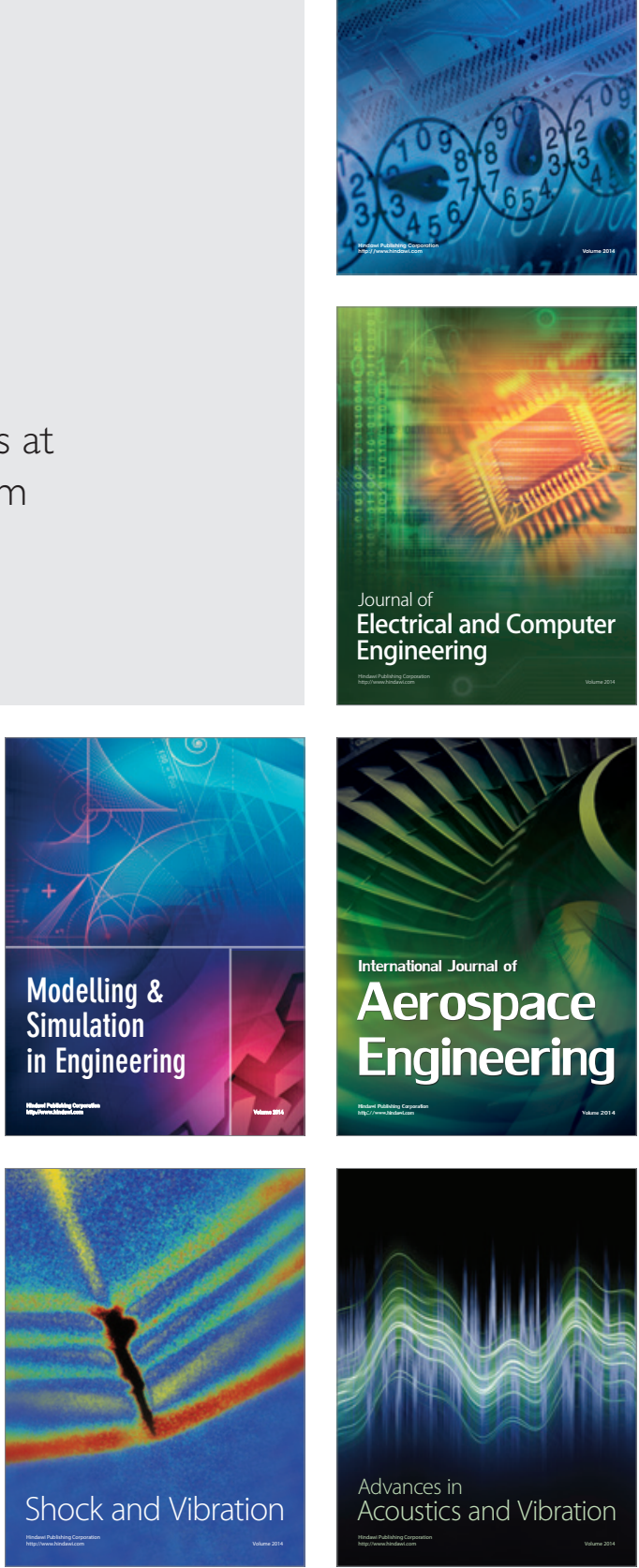